For completeness of statement, we note that the introduction to the second part is followed by a chapter of sixteen pages on complex numbers, containing De Moivre's theorem and the trigonometric solution of the binomial equation. There is also a chapter of six pages on elimination, with illustrations of Euler's and Sylvester's methods. The volume concludes with the methods of approximating to the real roots of numerical equations. Each theorem of the text, as far as possible, is immediately supplemented by problems showing its applications and place in the theory.

In conclusion, there are many admirable features in the work which would make it in some respects a valuable adjunct to instruction, and it is to be hoped that in a second edition a careful revision and the elimination of the rather numerous typographical errors will bring these qualities more clearly to light and place it in a position of considerable usefulness.

\title{
JAMES Maclay.
}

The Theory and Practice of Interpolation: Including mechanical quadrature and other important problems concerned with the tabular values of functions. With the requisite tables. By Herbert L. RICE, M.S. The Nichols Press, Lynn, Mass. 1899. Cr. 8vo., $234+$ ix pp.

THe theory and practice of interpolation is, in its essentials, based on the fact that in nearly all functions which arise in physical problems, a small change of the variable produces a small change of the function. The simplest case is the one in which we may consider the ratio of the two small changes as constant : in the language of the subject, the first differences are constant. When this assumption will not give sufficiently accurate results, we have to consider the difference of the first differences or the second differences, and even differences of higher order. Ultimately we neglect differences of some definite order and thus implicitly reduce the problem to the consideration of the values of a function which, between certain limits and to a given degree of accuracy, may be considered rational, integral and algebraic. Round this problem a mass of literature has grown up. The values of a function are required for certain values of the variable. To obtain these every time from a formula may be troublesome, or even impossible if no such formula is known ; tables are therefore made giving the values of the function for certain values of the variable, and the subject teaches how we can thence 
obtain an approximate value of the function for the given value of the variable, and conversely.

Mr. Rice, in this volume, appears to aim at placing before the reader all the formulæ which are likely to be required for computing purposes, rather than to give an account of the principles which underlie the methods used. He has developed many formulæ very fully so as to show how they may be applied to any particular case, and has illustrated their use by many numerical examples. The book is essentially a practical one and as such will doubtless be of great service to a student wishing to learn the methods in use, as well as to one who knows them but desires to have the results gathered together in one volume; and the tables at the end will greatly increase its value. To one interested in the subject from the mathematical point of view only, it will perhaps be found to be lacking in interest. But this separation of the theoretical from the practical may be necessary here if either is to be of service. From the practical side, the processes used carry their own justification, that is, the results appear to justify the means employed. The theorist would probably prefer to investigate their validity and determine their limitations. One of the chief problems for the latter is the consideration of the limits between which any of the functions which arise for consideration can be represented, either theoretically or usefully for computing purposes, by a rational integral function, and the degree of error committed in so doing.

There are three principal formulæ used-those of Newton, Stirling, and Bessel-which really amount to a development by Taylor's theorem, with the assumption that we can always stop at some particular term and neglect the remainder. The differences between the three formulæ simply consist in the manner in which the various orders of differences are grouped ; the best formula for any particular purpose is generally that which gives the required accuracy with the least amount of calculation. All these details are fully set forth by the author, and the reader will have no difficulty in immediately finding for practical use anything he may require.

One or two details call for slight criticism from the point of view of accurate statement. On p. 40 a remark is made which would leave the reader to infer that interpolation may give more accurate results than direct calculations even when both methods are available; this, if true, at least requires explanation. Again the author uses the elliptic integrals (with a real variable) as examples of non- 
integrable expressions when the modulus is near unity; this is misleading, as a transformation enables us in such a case to approximate as close as we wish to the numerical value of the integral. The writer of this notice has not tested any of the formulæ but they appear to be carefully worked out and the anthor seems to be familiar with the best methods of computation. ERNEST W. BRown.

Vorlesungen über Gesohichte der Trigonometrie. Von DR. A. voN Braunmühl. Erster Theil. Leipzig, B. G. Teubner, 1900. 260 pp.

VON BRAUNMÜHL's history of trigonometry is to be in two parts. The volume before us is the first part, and carries the narrative down to the invention of logarithms. If the second half of the work rises to the level of the first, the book will at once take its place as the most complete and authoritative work on the history of trigonometry.

In the perusal of this volume we realize the rapid progress which has been made in the study of mathematical history. The "restitution of decayed intelligence" has been carried on with great diligence. Formerly the origin of trigonometry was ascribed to Hipparchus and Ptolemaeus ; now the earliest attempts to establish relations between the sides and the angles of a right triangle can be traced back to a period as long before the time of Hipparchus, as Hipparchus was before our time. The Ahmes papyrus of about 2000 B. C. contains five problems involving such relations.

In Whewell's History of the inductive sciences we read of the "sterility of Arabian genius," but in recent years a closer acquaintance with Arabic research in mathematics forces us to the admission that the Arabs displayed considerable originality. Von Braunmühl makes it plain that in trigonometry much of what has been ascribed to Europeans of the fifteenth century was worked out at an earlier period by the Arabs. The author suggests that even our present knowledge may require considerable revision after Arabic manuscripts have been read, which at present, covered with the dust of centuries, lie untouched on the shelves of Spanish libraries.

The author has embodied in this volume a great deal of original research. He has brought to light the fact that the Greeks possessed a graphic method of solving spherical triangles which was extended by the Hindus and Arabs so as to become a fruitful aid in trigonometric computation. This 